\title{
Mean Free Path in Disordered Multichannel Tight-binding Wires
}

\author{
Jean Heinrichs* \\ Département de Physique, B5a, Université de Liège, \\ Sart Tilman, B-4000 Liège, Belgium
}

(Dated: May 4, 2018)

\begin{abstract}
Transport in a disordered tight-binding wire involves a collection of different mean free paths resulting from the distinct fermi points, which correspond to the various scattering channels of the wire. The generalization of Thouless' relation between the mean free path and the localization length $\xi$ permits to define an average channel mean free path, $\bar{\ell}$, such that $\xi \sim N \bar{\ell}$ in an $N$ channel system. The averaged mean free path $\bar{\ell}$ is expressed exactly in terms of the total reflection coefficient of the wire and compared with the mean free path defined in the maximum entropy approach.
\end{abstract}

PACS numbers: 73.23.-b,72.10.-d,72.15.Rn

*Electronic address: J.Heinrichs@ulg.ac.be 


\section{INTRODUCTION}

In a series of papers[1, 2, 3] (hereafter referred to as I, II, III) we studied localization for weak disorder in few channel $(N)$ wires, using a scattering matrix approach. The disordered wires in I and II were described by the Anderson tight-binding model and two- and three chain systems were considered where, for $N=3$, we further distinguished between chains arranged on a "tube" (periodic lateral boundary conditions) or on a "strip" (free lateral boundary conditions). The multichannel systems of length $N_{L}$ (in units of the lattice parameter $a$ ) were connected as usual to non-disordered leads.

In I we restricted to the familiar case where all channels are conducting at the fermi level. In II we extended our treatment to the case where one or several states at the fermi level are evanescent. In III we applied our treatment in the case of a model involving equivalent tight-binding chains with both random site energies and random interchain hoppings [4].

In addition to the localization length, $\xi$,we also studied elastic mean free paths, $\ell$, in II and III, using our scattering matrix results for averaged channel reflection coefficients. We used an expression for the mean free path in terms of the total reflection coefficient defined in the framework of a maximum entropy approach to multichannel conductors [5]. The maximum entropy approach has been further discussed in refs. 6,7 and has received considerable development as shown in extensive reviews [8, 9, 10, 11]. Unfortunately the mean free paths calculated from the expression proposed in [5, 6, 7, 8, 9, 10, 11], using our reflection coefficients [1, 2, 3], led to a very unsatisfactory general result [2, 3], namely

$$
\ell=\frac{\xi}{2}
$$

for all several-channel systems (and for various positions of the fermi level in each case) studied in I-III. Here $\xi$ refers to the exact weak disorder localization lengths obtained from the conductance in various cases[1, 2, 3]. The main problem of (11) is of course that its extrapolation to the many channel case would imply the non-existence of a mesoscopic metallic regime in a thick wire, in contrast to well-known results[12, 13]. This reveals an important insufficiency of the expression for the mean free path used as a constraint in the maximum entropy approach.

The purpose of this paper is to derive a new general expression of the mean free path which is rooted in the microscopic aspects of tight-binding wires such as those studied in 
I-III. The microscopic description leads us to define different mean free paths for the various conducting channels in a wire. From the individual channel mean free paths one may define an average channel mean free path which, in turn may be expressed in terms of the total reflection coefficient of the disordered wire. The use of this average mean free path expression palliates to the unsatisfactory features encountered with the mean free path expression of the maximum entropy approach. This is discussed in Sect. II below.

\section{MEAN FREE PATH FORMULA}

We first recall some relevant aspects of the study of the coupled tight-binding chain models of wires in I. In a first step of the analysis[1] the interchain hopping has been diagonalized in order to define new independent chains for the non-disordered leads. The Bloch wave solutions for these individual chains then define a set of independent channels for wave propagation and scattering by the disorder.The Bloch energy band associated with a channel $i$ in any of the few-channel systems studied in I is of the form

$$
E_{i}=\alpha_{i}-2 \beta \cos k_{i}
$$

where $-\beta$ is a constant hopping parameter between nearest-neighbour sites on the chain and $\alpha_{i}$ is related to a constant nearest-neighbour interchain hopping rate $h$. Specifically we have

$$
\alpha_{1}=0
$$

for a single-chain (one-dimensional) system,

$$
\alpha_{1}=h \quad, \quad \alpha_{2}=-h \quad,
$$

for a two-chain system $(i=1,2)$,

$$
\begin{aligned}
& \alpha_{1}=\sqrt{2} h \\
& \alpha_{2}=0 \\
& \alpha_{3}=-\sqrt{2} h,
\end{aligned}
$$


for a three-chain strip $(i=1,2,3)$ and, finally,

$$
\begin{aligned}
& \alpha_{1}=2 h \\
& \alpha_{2}=\alpha_{3}=-h,
\end{aligned}
$$

for a three-chain tube $(i=1,2,3)$.

Clearly for a wire composed of an arbitrary number $N$ of tight-binding chains the channelbasis band structure will include $N$ one-dimensional energy bands of the form(2). The allowed Bloch wavenumber values obtained by applying Born-von Karman boundary conditions are

$$
k_{i}=\frac{2 \pi m}{L}, m=0,1,2, \ldots
$$

where $L$ is the length of the wire.

In studying electrical transport in an $N$-channel wire with the band structure above we focus on typical situations where bands of the form(2) are mutually overlapping so that e.g. a number $N_{c} \leq N$ of the bands are conducting at the fermi level of energy $E_{F}$. This implies that the conduction electrons at the fermi level are distributed over $N_{c}$ distinct energy bands (2) at corresponding fermi points.

In this case the Drude conductivity $\sigma$ of the wire is given by 14$]$

$$
\sigma=e^{2} \sum_{i=1}^{N_{c}} \frac{n_{i} \tau_{i}}{m_{i}},
$$

where $n_{i}$ is the electron density in the $i$ th channel, $\tau_{i}$ is the relaxation time for electrons in channel $i$, and $m_{i}$ is the effective mass evaluated at the fermi level,

$$
m_{i}=\left.\frac{1}{\hbar^{2}} \frac{\partial^{2} E_{i}}{\partial k_{i}^{2}}\right|_{E_{i}=E_{F}} .
$$

We further define the electron velocity in the $i$ th branch, at the fermi level

$$
v_{F_{i}}=\left.\frac{1}{\hbar} \frac{\partial E_{i}}{\partial k_{i}}\right|_{E_{i}=E_{F}} .
$$

Now $n_{i}$ is given by $n_{i}=\frac{N_{i}}{A L}$ where $N_{i}$ is the total number of electrons in the band $E_{i}$ in the ground state and $A$ denotes the cross section area of the wire: $N_{i}$ is obtained by filling the $k$-levels with two electrons each up to the fermi level at $k_{F_{i}}$ given by 


$$
\cos k_{F_{i}}=\frac{E_{F}-\alpha_{i}}{2 \beta}
$$

From (10) we obtain $N_{i}=k_{F_{i}} L / \pi$. By inserting these results in (11) and defining the mean free path in the $i$ th channel, $\ell_{i}=v_{F_{i}} \tau_{i}$ we finally get

$$
\sigma=\frac{2 e^{2}}{h A} \sum_{i=1}^{N_{c}} \ell_{i}
$$

In mesoscopic systems one is primarily interested in the sample-specific conductance which, in the metallic regime to be identified, is given by Ohm's law, $g=\sigma A / L$. Using (15) we thus obtain

$$
g L=\frac{2 e^{2}}{h} \sum_{i} \ell_{i} .
$$

Now, as is well-known[15], the order of magnitude of the localization length $\xi$ is given by the range of lengths where the reduced or dimensionless conductance $g /\left(2 e^{2} / h\right)$ is of order one, since beyond that range the conductance decreases drastically (insulating regime). Thus from (16) we obtain the important result

$$
\xi \sim \sum_{i=1}^{N_{c}} \ell_{i}
$$

which proves the additivity of the localization length with respect to mean free paths in the individual channels. This shows in addition that the mesoscopic metallic domain extending over length scales from a typical mean free path up to the localization length will be the wider the larger the number of channels i.e. the thicker the wire.

It may be useful in practice to define an average mean free path over the fermi surface,

$$
\bar{\ell}=\frac{1}{N_{c}} \sum_{i} \ell_{i}
$$

of magnitude

$$
\bar{\ell}=\frac{\xi}{N_{c}}
$$

where we recall that $N_{c} \leq N$ is the number of conducting channels at the fermi level. In this case the range for the metallic domain reads 


$$
\bar{\ell} \ll l \ll N_{c} \bar{\ell}=\xi
$$

The use of a single elastic mean free path has been a common practice in the study of multichannel wires, starting with the influential paper of Thouless[12] on the proof of localization in such wires. In fact our analysis generalizes the discussion of Thouless in the case of a multichain tight-binding wire where the mean free paths in the conducting channels are generally different.

Finally, besides the definition (19) of an average mean free path, it is useful to obtain an exact expression of it in terms of scattering parameters, in analogy with a definition used in the maximum entropy approach [5, 6, 7, 8, 9, 10, 11]. We now derive such an expression for weak disorder. From (19) and the definition of the localization length from conductance (1) we have

$$
\frac{1}{N_{c} \bar{\ell}}=-\lim _{L \rightarrow \infty} \frac{\langle\ln g\rangle}{2 L},
$$

where $g$ is the Landauer two-probe conductance

$$
g=\frac{2 e^{2}}{h} \operatorname{Tr}\left(\hat{t} \hat{t}^{+}\right),
$$

$\hat{t}$ is the transmission amplitude matrix of the wire and $\langle\ldots\rangle$ means averaging over the disorder. From current conservation in an $N$-channel wire[2] it follow that

$$
\operatorname{Tr} \hat{t}^{+}=N-\operatorname{Tr} \hat{r} \hat{r}^{+},
$$

where $\hat{r}$ is the reflection amplitudes matrix. For weak disorder transmission coefficients are close to 1 and reflection coefficients are close to zero. Thus from (21-23) we obtain the exact expression

$$
\frac{1}{\bar{\ell}}=\frac{\left\langle\operatorname{Tr}\left(\hat{r} \hat{r}^{+}\right)\right\rangle}{2 L},
$$

for weak disorder and sufficiently large $L[16]$.

The essential difference between (24) and the expression for $1 / \ell$ used in the maximum entropy approach [5, 6, 6, 8, 9, 10, 11] lies in the presence in the latter of an additional factor of $1 / N$ (see e.g. (3.10) in Ref. 5). Returning to the detailed calculation of localization 
lengths and mean free paths in few channel systems in I-III we note that by replacing the mean free paths $\ell$ based on the maximum entropy expression (Eq. (57) in II) by the present correct result, $\bar{\ell}=\ell / N_{c}$, we obtain from (11), $\xi=2 N_{c} \bar{\ell}$ for all cases analyzed in I-III. This finally confirms that the coupled disordered tight-binding chain model provides a sound microscopic basis for describing multichannel wires and the corresponding mesoscopic metallic domain of these systems.

[1] J. Heinrichs, Phys. Rev. B 66, 155434 (2002).

[2] J. Heinrichs, Phys. Rev. B 68, 155403 (2003).

[3] J. Heinrichs, J. Phys.: Condens. Matter 15, 5025 (2003).

[4] O.N. Dorokhov, Phys. Rev. B 37, 10526 (1988).

[5] P.A. Mello, P. Pereyra and N. Kumar, Ann. Phys. NY 181, 290 (1988).

[6] P.A. Mello and B. Shapiro, Phys. Rev. B 37, 5860 (1988).

[7] P.A. Mello and A.D. Stone, Phys. Rev. B 44, 3559 (1991).

[8] A.D. Stone, P.A. Mello, K.A. Muttalib and J.L. Pichard, in Mesoscopic Phenomena in Solids (Edited by B.L. Altshuler, P.A. Lee and R.A. Webb, North Holland, Amsterdam, 1991).

[9] C.W.J. Beenakker, Rev. Mod. Phys. 69, 731 (1997).

[10] M. Janssen, Phys. Rep. 295, 1 (1998).

[11] P.A. Mello and N. Kumar, 2004, Quantum Transport in Mesoscopic Systems (Oxford University Press, Oxford).

[12] D.J. Thouless, Phys. Rev. Lett., 39, 1167 (1977).

[13] P.W. Anderson, D.J. Thouless, E. Abrahams and D.S. Fisher, Phys. Rev. B 22, 3519 (1981).

[14] A.H. Wilson, The Theory of Metals, second edition (Cambridge University Press, 1965).

[15] Y. Imry, Introduction to Mesoscopic Physics (Oxford University Press, New York, 1997).

[16] The mean free path is usually defined [5, 6, 7, 8, 9, 10, 11], following Anderson et al. [13], in terms of reflection from a small section of wire of length $\delta L$, whose resistance $\delta R=(\delta g)^{-1} \propto \delta L$ is assumed to be Ohmic. Since here we restrict to length scales (20) where the system is Ohmic we may replace $\delta L$ by $L$. 